\title{
An Ontology of Chinese Ceramic Vases
}

\author{
Tong Wei ${ }^{1,2} \mathbb{D}^{\mathrm{a}}$, Christophe Roche ${ }^{1,2 \mathbb{D}^{\mathrm{b}}}$, Maria Papadopoulou ${ }^{1,2} \mathbb{D}^{\mathrm{c}}$ and Yangli Jia ${ }^{2} \mathbb{D}^{\mathrm{d}}$ \\ ${ }^{1}$ Condillac Research Group of LISTIC Lab, University Savoie Mont-Blanc, Rue du Lac Majeur, Le Bourget du Lac, France \\ ${ }^{2}$ The School of Computer Science, Liaocheng University, HuNan Road 1, Liaocheng City, China
}

Keywords: Cultural Heritage, Ontology, Ontology Building, Semantic Web, Chinese Ceramic Vases.

\begin{abstract}
Extensive collections of Chinese ceramic vases are housed in museums throughout China. They could serve as rich sources of data for historical research. Although some data sources have been digitized, the vision of heritage institutions is not only to display objects and simple descriptions (drawn from metadata) but also to allow for understanding relationships between objects (created by semantically interrelated metadata). The key to achieving this goal is to utilize the technologies of the Semantic Web, whose core is Ontology. The focus of this paper is to describe the construction of the TAO CI ("ceramics" in Chinese) ontology of the domain of ceramic vases of the Ming (1368-1644) and Qing (1644-1911) dynasties. The theoretical and methodological approach adopted to construct the TAO CI ontology is term-and-characteristic guided, i.e., it relies on a morphological analysis of the Chinese terms used in the domain, and respects the ISO principles on Terminology (ISO 1087 and 704), according to which concepts are defined by means of essential characteristics. The research presented in this article aims to publish the resulting structured data on the Semantic Web for the use of anybody interested, including museums hosting collections of these vessels, and to enrich existing methodologies on domain ontology building. To our knowledge, there are no comprehensive ontologies for Chinese ceramic vases. TAO CI ontology remedies this gap and provides a reference for ontology building in other domains of Chinese cultural heritage. The tool used is Protégé. The TAO CI ontology is open access here: http://www.dh.ketrc.com/otcontainer/data/OTContainer.owl.
\end{abstract}

\section{INTRODUCTION}

China has a rich cultural heritage and has concentrated on producing digital data under the first wave of digitization. This is also true of the knowledge domain of Chinese ceramic vessels. The domain of Chinese ceramic vessels is rich, yet it lacks knowledge representation models (ontologies) to capture Chinese pottery concepts, express them in Semantic Web compatible interchange formats, and make them shareable and linkable to other data. As there is no ontology in the domain of Chinese ceramics providing the semantics of relevant data, most heritage institutions in China have not yet published cultural heritage data on the Semantic Web. Furthermore, every institution accumulates its data in its own traditional database system rather than linking data through an open data policy. To bridge this gap, this paper proposes the TAO CI ("ceramics" in Chinese) ontology. In compliance to the ethos of reuse recommended by the $\mathrm{W} 3 \mathrm{C}$, the $\mathrm{TAO} \mathrm{CI}$ ontology relates to existing ontologies and thesauri, such as CIDOC CRM (Cidoc, 2003), EDM (Doerr et al., 2010), and AAT (Soergel, 1995). First, the TAO CI ontology aims to provide an important reference for the publication of other cultural heritage ontologies and to be conducive to more and more Chinese heritage institutions publishing open cultural heritage data and linking them. Second, the theoretical and methodological approach adopted in the construction of the TAO CI ontology is term-andcharacteristic guided, i.e. it adopts the ISO principles on Terminology (ISO 1087 and 704), which define concepts on the basis of their essential characteristics for defining concepts. Last but not least, this work

a https://orcid.org/0000-0002-4159-6248

b(D) https://orcid.org/0000-0002-0756-0559

c(D) https://orcid.org/0000-0001-6451-8712

d(D) https://orcid.org/0000-0002-6849-1059 
aims at enriching existing methodologies of building domain ontologies and suggests that taking into account a term-and-characteristic guided approach, makes ontology engineering less dependent on formal languages and description logics as required background.

The rest of the paper is organized as follows: Section 2 describes the domain, section 3 introduces the objectives, section 4 is dedicated to the state of the art. Section 5 is the main section dedicated to our contribution to ontology building methodology relying on a morphological analysis of Chinese terms and on the ISO principles on Terminology. Section 6 presents the TAO CI ontology in Protégé, and the last section presents the evaluation of the TAO CI ontology.

\section{DOMAIN OF RESEARCH}

\subsection{Ming and Qing Dynasties}

Chinese history goes back about 5,000 years. Chinese ceramic vessels are among the most iconic objects of Chinese cultural heritage. Changes in pottery styles reflect the change in dynasties. In this paper, the focus is on Chinese porcelain vases defined as "clay vessels fired at high temperature used for decoration" of the Ming and Qing dynasties (冯先铭, 2002).

Ming Dynasty (1368-1644) ceramics were famous for the boldness of their form and decoration and the varieties of design. ${ }^{5}$ Already from the time of the Tang dynasty and the Song dynasty, there were many famous kilns and many different types of ceramic vessels. From the beginning of the Ming dynasty, the Jingdezhen kiln gradually became the most important production place: ceramic vessels of the Jingdezhen kiln represented the highest quality at the time. Between 1350 and 1750, Jingdezhen was a center of production for nearly all of the world's porcelain.

Qing dynasty (1644-1911) porcelain was famous for its polychrome decorations, delicately painted landscapes, and bird and flower as well as multicolored enamel designs. The peak of Chinese ceramics production took place in the reigns of Kangxi (1661-1722), Yongzheng (1722-1735), and Qianlong (1735-1796) during which improvement

\footnotetext{
${ }^{5}$ http://factsanddetails.com/china/cat7/sub40/item258.html \#chapter-11

${ }^{6} \mathrm{https} / / /$ www.comuseum.com/ceramics/qing/

${ }^{7}$ https://www.dpm.org.cn/Home.html

${ }^{8} \mathrm{http} / / /$ www.chnmuseum.cn/
}

was seen in almost all ceramic types, including the blue and white wares, polychrome wares, monochrome wares, etc. ${ }^{6}$ During the Qing dynasty, potters began using bright colors to adorn plates and vases with meticulously painted scenes.

\subsection{Collection of Vases}

The first step of our work was to select the set of vases to study. The set had to be enough representative of the richness of the domain without being too big since the main and first goal was defining the ontology rather than populating it. In China, many museums publish much information about ceramic vessels on their websites. One hundred forty-nine objects were selected from different museums in China. Ninetyseven objects come from the Palace Museum ${ }^{7}$ that has the most important collection of ceramics. Twenty-two objects come from the National Museum of China ${ }^{8}$, twenty-four objects from the Guangdong Museum ${ }^{9}$. Four objects come from the Shanghai Museum ${ }^{10}$ and two objects from the Capital Museum $^{11}$. For the selection of objects, we have adopted the following three criteria. The two first concern the selection of the museum, which had to fulfil the following conditions: first, the collection of ceramics had to be recognized as a reference in ceramic vessels in China; second, the information about the collection should be publicly available and precise enough for the building of an ontology. The third principle was to select objects as different as possible, i.e., of different types according to their shape, the technique of making, decoration, etc.

\section{OBJECTIVES}

The TAO ontology has two aims. The first one is to build a knowledge representation of Chinese ceramic of the Ming and Qing dynasties in the form of an open ontology. The second one is to provide a bilingual (Chinese-English) e-dictionary of ceramics vases. The competency questions (Ren et al., 2014) that were used to specify the requirements of the ontology are shown in Table 1.

\footnotetext{
${ }^{9} \mathrm{http} / / / \mathrm{www} . \mathrm{gdmuseum} . c 0 \mathrm{~m} /$

${ }^{10} \mathrm{https}: / /$ www.shanghaimuseum.net/museum/frontend/

${ }^{11} \mathrm{http}: / /$ www.capitalmuseum.org.cn/
} 


\section{STATE OF THE ART}

The state of the art presented here features work done in an effort to produce interoperable vocabularies for the expression of Cultural Heritage data. It includes W3C languages, semantic data models, thesaurus and ontology resources, and ontology building methodologies.

Opening cultural heritage on the web relies on W3C standards such as OWL ${ }^{12}$ (Web Ontology Language), a Semantic Web language designed to represent rich and complex knowledge about things, groups of things, and relations between things, SKOS $^{13}$ (Simple Knowledge Organization System), a data model for sharing and linking knowledge organization systems on the Web. SKOS can be used to capture much of the semantics of existing thesaurus of museums and other memory institutions thesauri. Let us also quote DC (Dublin Core), a metadata schema based on 15 essential properties to describe online and physical resources ${ }^{14}$.

Semantic Data Models for the Cultural Heritage domain have to be taken into account. In particular, CIDOC-CRM, a meta-ontology for the representation of concepts for the use of museum and cultural heritage specialists (Cidoc, 2003). It provides a semantic framework to building a mapping between different cultural heritage resources reducing their heterogeneity (Doerr, 2003). Our work not only aims to build an ontology for museum publishing open museum data, but also aims to build a multilingual terminological knowledge base. From a terminological point of view, we need to build a more 'granular' ontology for knowledge representation of Chinese ceramic vases. Let us also quote EDM, the common data model that was built in order to harmonize data from different providers of Europeana (Doerr et al., 2010). It is used for the representation of concepts in the cultural heritage domain. It is not a fixed schema that dictates the way of representing data, but rather a conceptual framework (or ontology) to which more specific models can be attached, and interoperability between them enhanced.

As far as ontological resources that the TAO CI project can benefit, let us quote AAT (The Art \& Architecture Thesaurus), a structured resource that can be used to improve access to information about

\footnotetext{
12 https://www.w3.org/TR/2004/REC-owl-features-20040210/

${ }^{13}$ https://www.w3.org/TR/skos-reference/\#notes

${ }^{14} \mathrm{https}$ ///dublincore.org/schemas/ Schemas are machineprocessable specifications that define the structure and syntax of metadata specifications in a formal schema language
}

art, architecture, and other material culture through rich metadata and links, hoping to provide (along with other Getty vocabularies) a powerful conduit for research and discovery in digital art history and related disciplines ${ }^{15}$ (Soergel, 1995). The AAT comprises over 250,000 terms on architectural history, styles, and techniques. Our ontology has been linked with AAT in order to provide more information for our terms in the ontology. Kerameikos ${ }^{16}$ is a "collaborative project dedicated to defining the intellectual concepts of pottery following the tenets of linked open data and the formulation of an ontology for representing and sharing ceramic data across disparate data systems." (Gruber \& Smith, 2014). Let us also quote Ontoceramic, which is an OWL ontology for ceramics classification (Cantone et al., 2015). Lekythos ${ }^{17}$ is an another project that aims at representing concepts in the domain of ancient Greek pottery, but having natural language terms in the domain as its starting point.

According to (Ushold, 1998), "An [explicit] ontology may take a variety of forms, but necessarily it will include a vocabulary of terms and some specification of their meaning (i.e., definitions)." For domain experts, identifying and defining concepts in ontology also presents a challenge for which ontology building methodology can bring useful aids. Ontology building methods can be based on objective criteria, e.g., clarity, coherence, extensibility, etc. (Gruber, 1995), software engineering methods (Fernández-López, 1999), text-based construction (Zouaq \& Nkambou, 2009), modular design approach (Özacar et al., 2011), ontological engineering (Suárez-Figueroa et al., 2012), unsupervised domain ontology learning method (Venu et al., 2016) , based on Formal Concept Analysis (Nong et al., 2019), etc. Let us quote some methodologies focusing on the stages which compose them. METHONTOLOGY (Fernández-López et al., 1997) includes seven stages: specification, knowledge acquisition, conceptualization, integration, implementation, evaluation, and documentation. On-To-Knowledge Methodology (Sure et al., 2004) includes the following phases: feasibility study, kick-off, refinement, evaluation, and application \& evolution.

$\mathrm{NeOn}$ methodology (Suárez-Figueroa et al., 2015) provides nine scenarios for developing ontologies.

\footnotetext{
15 https://www.getty.edu/research/tools/vocabularies/aat/ about.html

16 http://kerameikos.org/

${ }^{17}$ http://o4dh.com/lekythos
} 
Table 1: The competency questions.

\begin{tabular}{|c|c|c|c|}
\hline CQ & Competency Question & Class & Relation \\
\hline 1 & What are the different types of vase? & Vase & vase-type is-a Vase \\
\hline 2 & What material is vase the made of? & Vase, material & Vase is made of Material \\
\hline 3 & What is the color and glaze of the vase? & Vase, Glaze-color, & Vase glaze-color is \\
\hline 4 & Which dynasty is the vase? & Vase, Dynasty & Vase hasDynasty Dynasty \\
\hline 5 & Which emperor is the vase? & Vase, Emperor & Vase hasEmperor Emperor \\
\hline 6 & What are the Chinese and English terms of & Vase & Vase label string \\
\hline 7 & What are the components of the vase? & Vase, Component & Vase hasComponent Component \\
\hline 8 & What is the function of the vase? & Vessel, Function & Vessel hasFunction Function \\
\hline 9 & Which dynasty does the emperor belong & Emperor, Dynasty & Emperor belongTo dynasty \\
\hline 10 & Where is the vase $\mathrm{x}$ collected in? & Vase $\mathrm{x}$ & Vase $\mathrm{x}$ is Collected In string \\
\hline 11 & Which kiln is the vase $\mathrm{x}$ produced? & Vase $\mathrm{x}$ & Vase $\mathrm{x}$ is produced in string \\
\hline 12 & What is vase $\mathrm{x}$ decorated with? & Vase $\mathrm{x}$ & Vase $\mathrm{x}$ is decorated by string \\
\hline 13 & What is the image of vase $\mathrm{x} ?$ & Vase $\mathrm{x}$ & Vase $\mathrm{x}$ image string \\
\hline
\end{tabular}

\section{TAO CI METHODOLOGY}

Ontology building follows a lifecycle made up of several stages (Fernández-López et al., 1997). Some of them have to be specialized, and others have to be introduced to take into account the specificities of the domain. The theory of concept underpinning the ontology can also strongly impact the building methodology. Following the ISO principles on Terminology where "a term is a verbal designation of a concept" and "a concept is a unique combination of (essential) characteristics ${ }^{18 "}$ (ISO 1087, ISO 704), we were led to adopt a "term-and-characteristic" guided methodology derived from works carried out in Digital Humanities (Roche \& Papadopoulou, 2019). Identifying essential characteristics becomes the main goal to achieve.

The problem of identifying essential characteristics, is a new and central phase of our methodology. This phase is aimed both at identifying differences between objects (vases with neck versus vases without neck) and on a morphological analysis of Chinese terms whose characters carry meaning in relation to the denoted objects, e.g. the term "清德化 窑白釉堆雕花卉瓷碗" where the first character (清) which represents the Qing dynasty and the last one the type of vase (碗).

18 An "essential characteristic" is a characteristic (abstraction of a property) of a concept and is indispensable to understanding that concept (ISO 1087). Essential characteristics correspond to rigid predicates in DL(Guarino \& Guizzardi, 2006) and to rigid properties in
The term-and-characteristic guided methodology includes seven steps. Each of them aims at different tasks: step 1: identify the scope of the domain and the objectives, step 2: identify terms and objects, step 3 : identify essential characteristics, step 4: define concepts, step 5: build ontology using one of the available tools, step 6: integration of other resources, step 7: evaluation.

\subsection{Identifying Essential Characteristics}

There are two approaches to identifying essential characteristics. The first one relies on identifying differences between objects, e.g., in their structure: vase with or without neck. The second one is based on a morphological analysis of Chinese terms whose characters directly express knowledge about the denoted objects. We will adopt the following notation: essential characteristics will be enclosed between slashes, e.g. /garlic shape mouth/.

\subsubsection{Differences between Objects}

Identifying differences between objects is a useful means towards identifying essential characteristics. The differences can be functional (e.g., vase for

the OntoClean method (Guarino \& Welty, 2004). Unlike essential characteristics, which define the concept, "descriptive characteristics" own values which describe the current state of an object, e.g. weight, colour, etc. 
decoration, vase for storing), material (in clay, in bronze), structural (with or without foot). Thus, one can rely on the part-of relationship between a whole and its parts to understand the concept the object belongs to (Gerstl \& Pribbenow, 1996). The presence or the absence of a component can be interpreted as essential characteristics. For example, a Chinese ceramic vase has a lid, a mouth, a neck, handle, shoulder, belly, and foot (Figure 1).
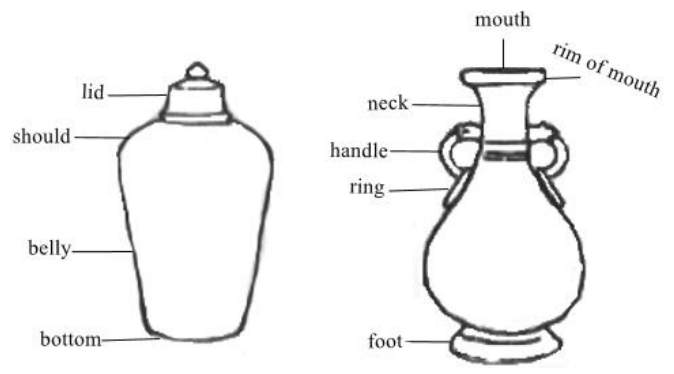

Figure 1: Parts of a vase.

From the handle point of view, vases can be split into vases with handles and vases without handles corresponding to the essential characteristic /with handle/ and /without handle/. The type of vases with handles can be itself specialised according to the different shapes of handles defining as many as corresponding essential characteristics: /dragon-mask handle/, /dragon-shaped handle/, /elephant-shaped handle/, /fish-shaped handle/, /halberd shaped handle/, /phoenix shaped handle/, /pierced handle/, /ribbon shaped handle/, and /Ru-Yi handle/ (Figure 2 ). These characteristics are exclusive to each other.

\subsubsection{Morphological Analysis of Chinese Terms}

The characters which compose the Chinese terms of vase carry important information about the nature and the description of the objects denoted by the terms.
Terms are composed of a set of characters of which the last one corresponds to the type of vase and the others, called modifiers, precise the type. For example, the Nanjing museum adopts the following order of modifiers for naming Tibetan ceramic (霍华, 1989): dynasty + kiln + glaze + colour + decoration + shape + texture + type. The information conveyed by the modifiers expresses knowledge of different nature, either essential, such as shape, material, and type, or descriptive, like glaze and color. For example, the term “清 雍正 粉青釉 凸花 如意耳 蒜 头瓷 瓶” (for convenience of non-Chinese speaker, we put spaces between modifiers) conveys the descriptive characteristics of dynasty (“清” Qing dynasty), emperor (“雍正” Yongzheng mark), glazecolor (“粉青釉” powder blue glaze), and decoration (“ 凸花” designed with flowers). It also conveys the essential characteristics of handle (“如意耳” Ru-Yi handle), shape (“蒜头” garlic-like head), material (“ 瓷” porcelain), and type (“瓶” vase). The English translation of the Chinese ceramic terms used by the Nanjing museum does not follow the Chinese order of modifiers, but the following order: glaze + colour + shape + texture + type + decoration + period + kiln. Thus, the previous term “清 雍正 粉青釉 凸花 如意 耳 蒜头瓷 瓶” is translated as: “powder blue glaze garlic porcelain vase designed with flowers and $\mathrm{Ru}$ Yi handles, the Yongzheng mark of Qing dynasty". This object belongs to the type of Garlic-head Vase (“蒜头瓶”).

\subsection{Combining Essential Characteristic}

From the ISO point of view on terminology, a concept is defined as a unique combination of essential characteristics (ISO 1087). Nevertheless, not any combination of essential characteristics defines a meaningful concept from the expert's point of view.

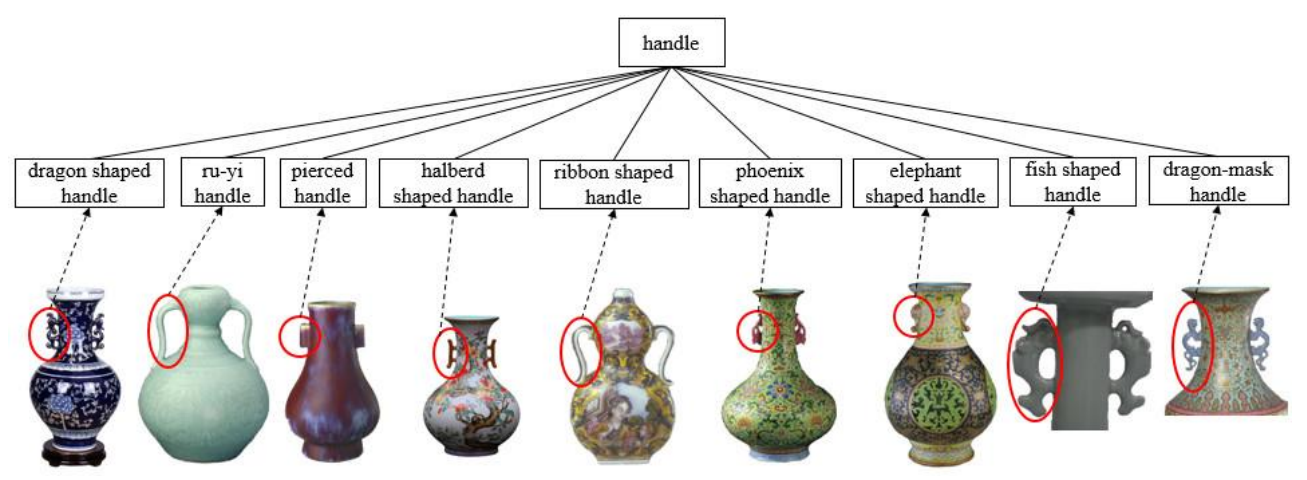

Figure 2: The essential characteristics of the analysis axis of the handle shape. 
For the expert, concepts of interest are those that are named in a natural language. Hence, a concept is a set of essential characteristics stable enough to be named in a given language (even if some concepts, without any designation in natural language, can be introduced for organisational purposes of the conceptual system). Terms can be then considered as guidelines for identifying domain concepts to be defined from the expert point of view. For example, the Chinese term “蒜头瓶”, "garlic vase" in English, denotes the following set of essential characteristics \{/vase/, /one mouth/, /garlic shape mouth/, /ring foot/\}. Based on this formal definition, the definition in natural language is then: "Vase with a garlic shape mouth with a ring foot". We notice that the characters “圈足” (“ring foot") do not appear in the name of the concept, an ellipsis owed to the fact that both types of garlic vase (Garlic vase I and Garlic vase II) have a ring foot.

\subsection{Implementation}

The following will present the implementation in Protégé of our ontology building approach. Concepts are represented as named classes in Protégé, and objects as individuals. Terms are represented as labels (skos:prefLabel, skos:altLabel, skos:definition). Relations, e.g. 'hasFunction', 'hasComponent', 'isMadeOf', are represented as object properties. For example, the object property 'isMadeOf' whose domain is the Vessel class and range is the Material Class, and the object property 'hasComponent' whose domain is the Vessel class and range is the Component class. Let us note that among the different types of 'part-of' relationships, only the 'Component/Integral Object' relationship has been taken into account (Winston et al., 1987). Descriptive characteristics are attributes whose values describe the current state of an object. The TAO CI ontology includes 10 descriptive characteristics: dynasty, emperor, kiln, color and glaze, height, diameter of mouth, diameter of foot, decoration, museum, and image. The descriptive characteristics are represented either as data properties, if their value is a data literal, or as object properties and classes, if the value is an individual. For example, the decoration characteristic is represented by the data property 'isDecoratedBy' whose domain is the Vessel class and the range is the String data type. The dynasty to which a vase belongs is represented by the object property 'hasDynasty'

19 The ontology file is published on http://www.dh.ketrc.com/otcontainer/data/OTContainer.o wl whose domain is the Vessel class and the range is the Dynasty class.

Implementing essential characteristics is a slightly more complex process. Since essential characteristics correspond to rigid predicates (Guarino \& Guizzardi, 2006) they cannot be directly expressed into Description Logic. Essential characteristics are expressed as classes. Thus, essential characteristics corresponding to parts of vase are subclasses of the Component class: Lid class, Mouth class, Neck class, Handle, Shoulder, Belly, Foot class, etc. Some being themselves specialized into subclasses according to the different types of parts: LongNeck and ShortNeck subclasses of the Neck class, RingFoot and SquareFoot subclasses of the Foot class, etc. Essential characteristics corresponding to functions, such as /for decoration/ are subclasses of Function class, etc. Owning an essential characteristic for a concept (class) is represented as a restriction of an object property whose range is the class associated to the essential characteristic. It means that the class (concept) is a subclass of the anonymous class defined by the restriction (see figure 5). For example, owning the essential characteristic /long neck/ will be translated into the following restriction of the 'has_component' object property: 'has_component' some LongNeck. The following restriction of the 'has_function' object property: 'has_function' some FunctionForDecoration expresses the owning of the essential characteristic/for decoration/.

Protégé relies on the open-world assumption, which means that what is not known to be true is unknown. In this vein, it is necessary to express information corresponding to essential characteristics such as /without handle/, /without lid/, /without foot/, etc. The object property restriction allows to do that. For example, owning the /without handle/ essential characteristic will be translated into the following object property restriction not ('has_component' some Handle).

\section{TAO CI ONTOLOGY}

The TAO CI ontology ${ }^{19}$ contains 165 classes, 11 object properties, 8 data properties, 132 individuals, and 3124 axioms. It is mapped with CIDOC CRM ${ }^{20}$ (E4_Period, E21_Person, E22_Man-Made_Object, E57_Material) and AAT.

\footnotetext{
${ }^{20} \mathrm{http} / / /$ www.cidoc-crm.org/cidoc-crm/
} 


\subsection{Class}

Although our work focuses on Chinese ceramic vases, The TAO CI ontology also includes other types of vessels, such as Box, Bowl, Jar (Figure 3). The Vase class is the core of our work. It includes 41 subclasses representing 41 different types of Chinese ceramic vases (Figure 5).

Let us consider the following example. The concept denoted by the term "arrow vase I", whose definition in natural language is "vase with a square mouth, long neck, slanting shoulder, pierced handle, bulge belly, and square foot", is defined by the set of essential characteristics: [/vase/, /square mouth/, /long neck/, /slanting shoulder/, /pierced handle/, /bulge belly/, /square foot/\}. It is represented by the ArrowVase_I class defined in OWL as subclass (rdfs:subClassOf) of:

- ArrowVase

- hasComponent some SquareMouth

- hasComponent some LongNeck

- hasComponent some SlantingShoulder

- hasComponent some PiercedHandle

- hasComponent some BulgeBelly

- hasComponent some SquareFoot

\subsection{Property}

Object properties include belongTo (domain:Emperor, range: Dynasty), hasFunction (domain:Vessel, range:Function), hasComponent (domain:Vessel, range: Component), etc.

Data properties include isDecoratedBy, diameterOfFoot, height, isProducedIn, diameterOfMouth, mouthNumber, etc.

\subsection{Annotations}

Annotations allows to enrich the description of the ontology and thus facilitate its understanding and reuse. The RDFS, DC, and SKOS vocabularies are used to express metadata and the linguistic dimension associated to a concept (dc:publisher, dc:license, dc:creator, skos:prefLabel, skos:altLabel, skos:definition, rdfs:comment) as well as to express linking and mapping to external resources (rdfs:seeAlso, skos:broadMatch, skos:exactMatch). For example, the individual arrow vase 001 is described as follows (Figure 4).

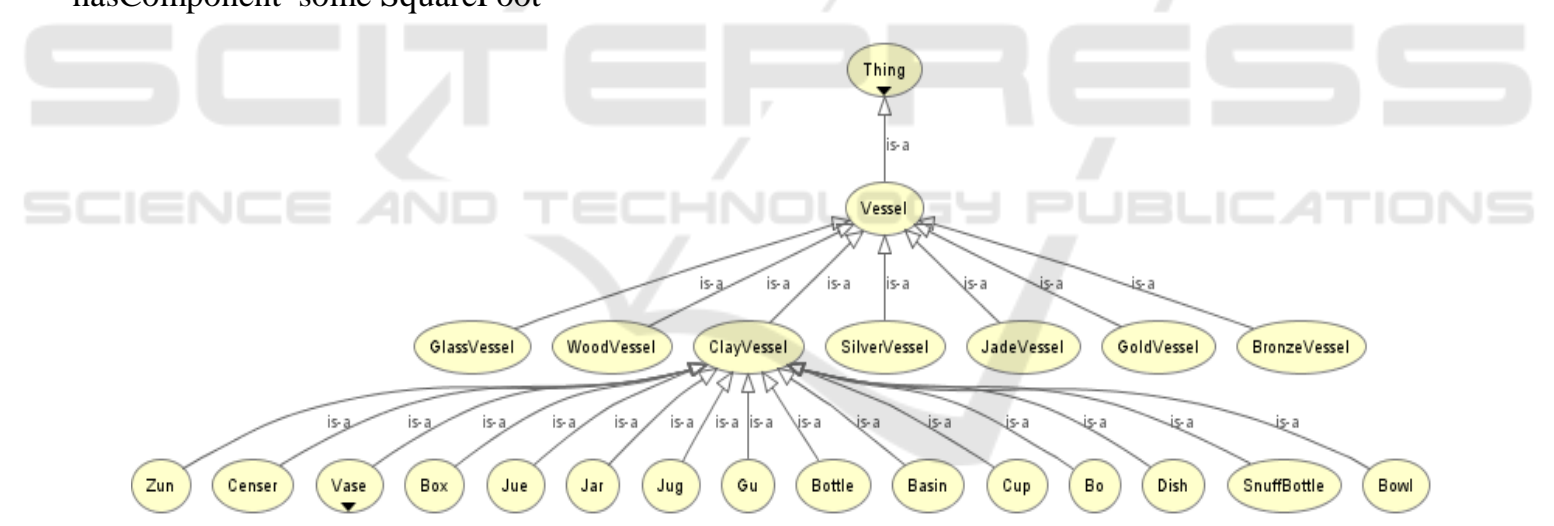

Figure 3: The Vessel class of TAO CI ontology.

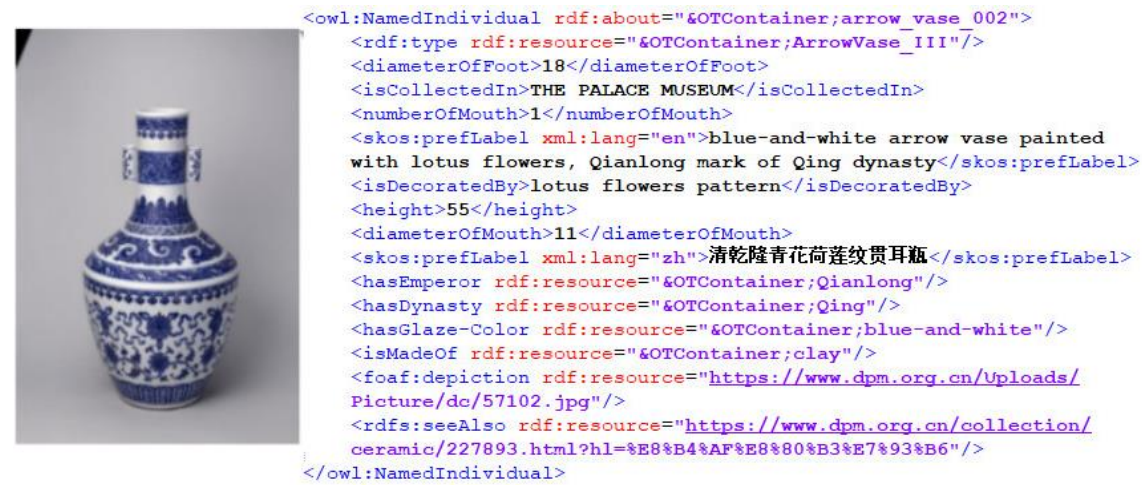

Figure 4: The individual arrow vase 001. 


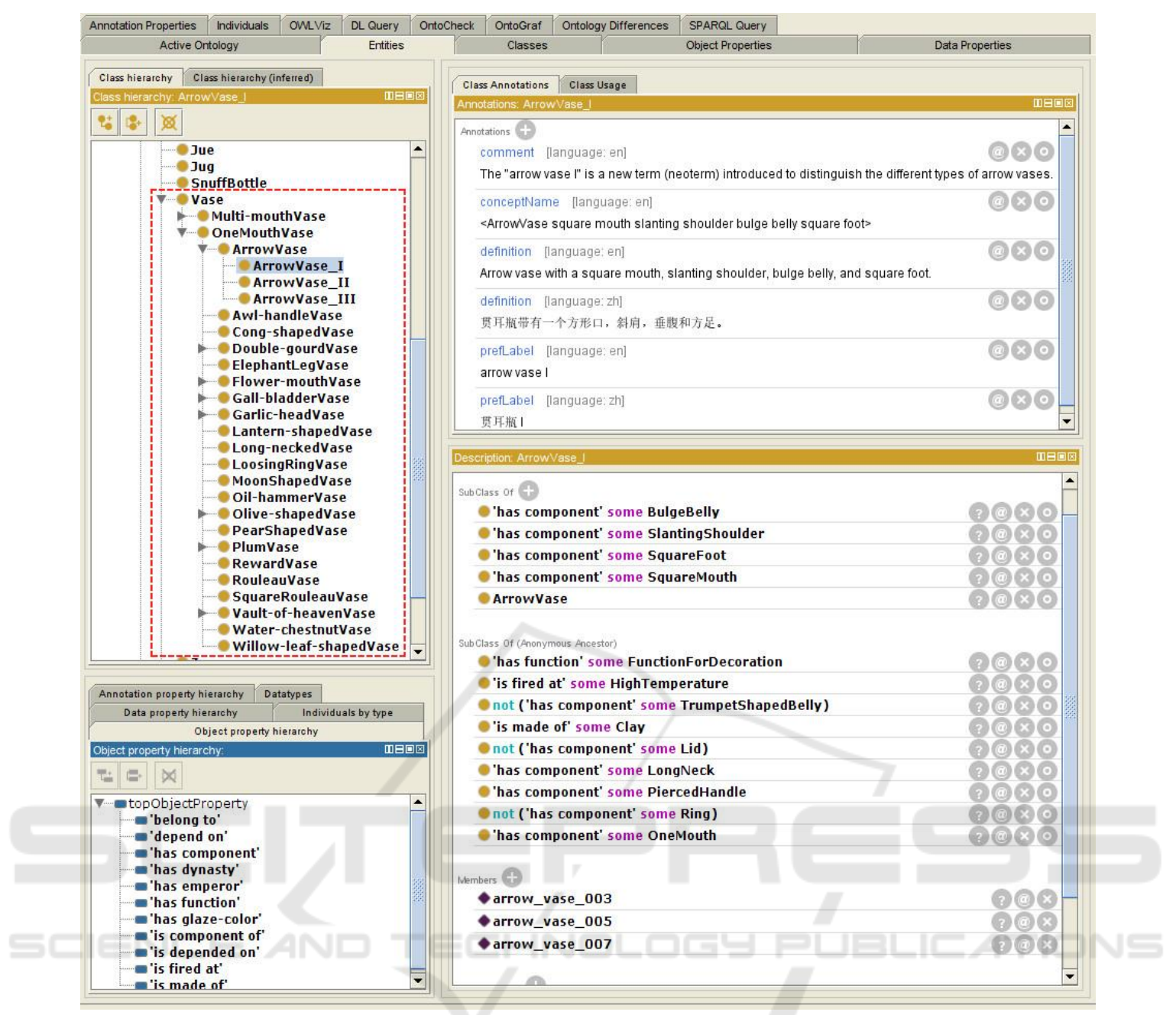

Figure 5: The Vase classes.

\section{EVALUATION}

The last stage of ontology building is ontology evaluation whose main goal is "to assess the quality and correctness of the obtained ontology" (Sabou \& Fernandez, 2012). We used two online platforms and queried the ontology against the Competency Questions defined in chapter 3.

The TAO CI ontology was submitted to OOPS!, an online tool to detect some of the most common pitfalls appearing when developing ontologies (Poveda-Villalón et al., 2014). OOPS! has detected only minor pitfalls for the TAO CI ontology (e.g. P08 "Missing annotations", P13 "Inverse relationships not explicitly declared").

\footnotetext{
${ }^{21} \mathrm{https} / / /$ ontometrics.informatik.uni-rostock.de/wiki/in dex.php/Schema_Metrics
}

The TAO CI ontology was also submitted to OntoMetrics, an online platform to calculate more advanced ontology metrics (Lantow, 2016). The table 2 shows some schema metrics and knowledge base metrics results ${ }^{21}$ in relation to ontology clarity and conciseness (Denny, 2010).

Table 2: TAO CI advanced metrics.

\begin{tabular}{|c|c|}
\hline Metric & Value \\
\hline Attribute richness & 0.048485 \\
\hline Inheritance richness & 2.715152 \\
\hline Relationship richness & 0.334324 \\
\hline Class/Relation ratio & 0.245171 \\
\hline Average population & 0.8 \\
\hline Class richness & 0.321212 \\
\hline
\end{tabular}


Most of the scores are very low. That is due to:

- the implementation of essential characteristics in Description Logics. Essential characteristics are translated as classes without any attributes (attribute richness);

- the main goal of the TAO CI ontology is the classification of vases; neither to represent relationships between vases and other artefacts (relationship richness, class/relation ratio), nor to populate the ontology with individuals (average population, class richness).

Evaluation of criteria strongly depends on the aims of the ontology and the choices made in to regard to its implementation: "a good ontology does not perform equally well with regards to all criteria" (Denny, 2009).

Let us also note that, in regard to our objectives of classification and terminology, the TAO CI ontology well covers the domain in the sense that each individual clearly falls into a concept (classification), and each concept is clearly defined as a unique combination of essential characteristics (terminology).

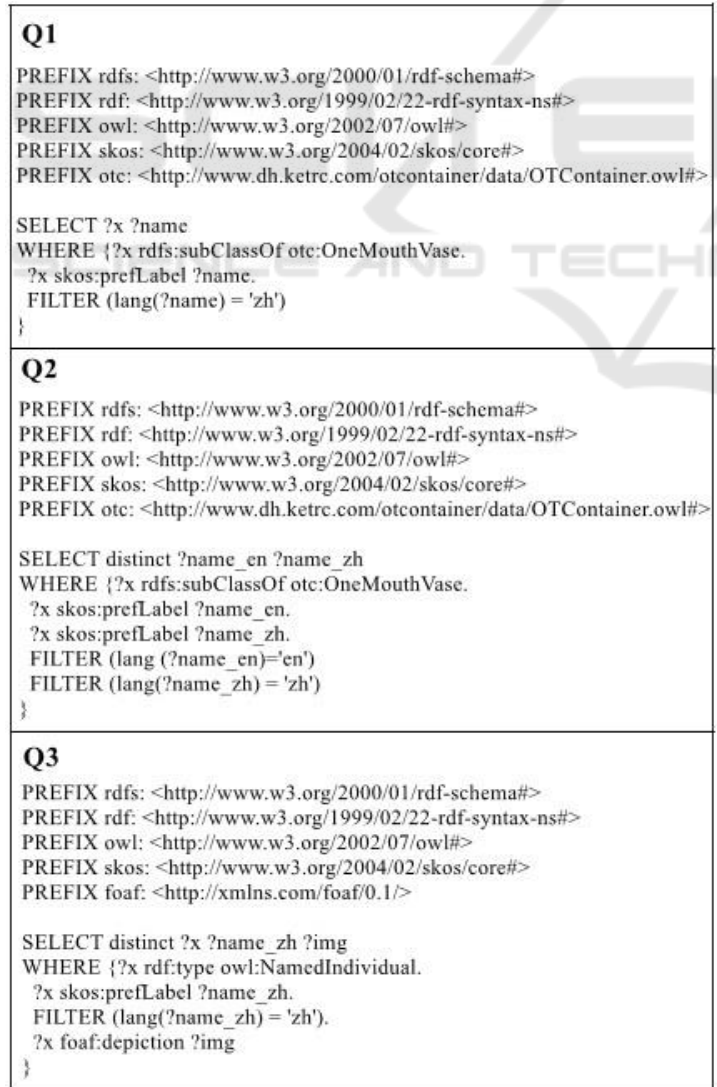

Figure 6: The competency questions expressed in SPARQL.
The last validation concerns the answers to the Competency Questions. All of them are satisfied. Figure 6 presents 3 competency questions translated into SPARQL and figure 7 the returned results.

Q1: What are the types of a vase?

Q2: What are the Chinese terms and the English terms for subclasses of the class Vase?

Q3: What are the Chinese terms, the English terms, and the images of individuals?

\section{CONCLUSION}

The objectives of the TAO CI project are to provide, first, a knowledge representation of Chinese ceramic of the Ming and Qing dynasties in the form of a domain ontology to be published in the LOD, and, second, a bilingual (Chinese-English) e-dictionary of vases based on the domain ontology. We adopted a term-and-characteristic guided methodology derived from taking into account the ISO principles on Terminology whereby "a term is a verbal designation of a concept" and "a concept is a unique combination of (essential) characteristics".

In order to identify essential characteristics, the core task of our ontology building methodology, we proposed to combine two approaches. The first one is object-oriented. It consists of identifying differences between objects, either structural or functional. The second one is language-dependent. It relies on a morphological analysis of Chinese terms whose characters convey a lot of useful semantic information about vases.

The implementation of the TAO CI ontology in Protégé raised the problem of how to implement the notion of essential characteristic. Since essential characteristics cannot be directly expressed in Description Logics, we proposed to represent them as classes. Owning an essential characteristic for a concept (class) is then represented as a restriction of an object property whose range is the class associated to the essential characteristic. This means that the concept (class) is a subclass of the anonymous class defined by the restriction.

The result is an open RDF/OWL ontology accessible from the web site http://www.dh.ketrc.com/. A bilingual (ChineseEnglish) dictionary based on the TAO CI ontology is also accessible from the same web site.

Further work is to be carried out in two different directions. The first one consists in enriching the linguistic dimension. Currently terms are reduced to labels on class, some vocabularies such OntoLexLemon would allow to represent the linguistic 


\begin{tabular}{|l|l|}
\hline \multicolumn{1}{|c|}{ name_en } & \multicolumn{1}{|c|}{ name_zh } \\
\hline "arrow vase"@en & "贯耳瓶"@zh \\
\hline "double-gourd vase"@en & "葫芦㼛"@zh \\
\hline "gall-bladder vase"@en & "胆式瓶"@zh \\
\hline "garlic-head vase"@en & "蒜头㼛"@zh \\
\hline "olive shaped vase"@en & "檄笕瓶"@zh \\
\hline "plum vase"@en & "梅瓶"@zh \\
\hline
\end{tabular}

Result of the Q1

\begin{tabular}{|c|c|}
\hline $\mathrm{x}$ & name \\
\hline http://www.dh.ketrc.com/otcontainer/data/OTContainer.owl\#ArrowVase & "贯耳臨"@2h \\
\hline http://www. dh ketrc.com/otcontainer/data/OTContainer.owl \#DoubleGourdShapedVase & "葫芦撽"@zh \\
\hline http://www. dhaketrc.com/otcontainer/data/OTContainer.owlitGall-bladderVase & 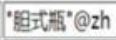 \\
\hline http://www.dh_ketrccom/otcontainer/data/OTContainer.owl\#Garlic-headVase & "萚头伍"@zh \\
\hline http://www. dh.ketrc.com/otcontainer/data/OTContainer.owl\#Olive-shapedVase & 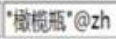 \\
\hline http://www dh ketrc com/otcontainer/data/OTContainer.owl\#PlumVase & “海留”@zh \\
\hline
\end{tabular}

Result of the Q2

\begin{tabular}{|c|c|c|}
\hline $\mathrm{http}: / /$ www dh ketrccom/otcontainer/data/OTContainerowl\#arrow_vase 001 & 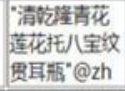 & https:/www.dpm.org.cn/Uploads/Picture/dc/3664.jpg \\
\hline http://uww.dh.ketrc.com/otcontainer/data/OTContainer.owl & 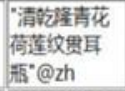 & https://www.dpm.org.cn/Uploads/Picture/dc/57102.jpg \\
\hline http://uww.dh.ketrc.com/otcontainer/data/OCContainer.owl\#arrow_vase_003 & $\begin{array}{l}\text { "清宣统乐青 } \\
\text { 种杏圆 } \\
\text { 非"@zh }\end{array}$ & https://www.dpm.org.cn/Uploads/Picture/dc/16328.jpg \\
\hline
\end{tabular}

Result of the Q3

Figure 7: An excerpt of the results.

dimension. The second one aims to complete the ontology by taking into account additional types of ceramic vessels.

\section{ACKNOWLEDGEMENTS}

This work has received generous funding by the China Scholarship Council (CSC) in the framework of a $\mathrm{PhD}$ program between Liaocheng University and University Savoie Mont-Blanc.

\section{REFERENCES}

Buitelaar, P., Cimiano, P., \& Magnini, B. (2005). Ontology learning from text: methods, evaluation and applications (Vol. 123). IOS press.

Cidoc, C. (2003). The CIDOC Conceptual Reference Model.

Corcho, O., Fernández-López, M., \& Gómez-Pérez, A. (2003). Methodologies, tools and languages for building ontologies. Where is their meeting point? Data \& Knowledge Engineering, 46(1), 41-64.

Cantone, D., Asmundo, M. N., Santamaria, D. F., and Trapani, F. (2015). Ontoceramic: an owl ontology for ceramics classification. In CILC, pages 122-127.

Denny, V. (2010). Ontology Evaluation. PhD thesis, Karlsruhe Institute of Technology.

Denny, V. (2009). Ontology evaluation. In Handbook on ontologies (pp. 293-313). Springer, Berlin, Heidelberg.

Doerr, M. (2003). The CIDOC Conceptual Reference Module: An Ontological Approach to Semantic Interoperability of Metadata. AI Magazine, 24(3), 7575. https://doi.org/10.1609/aimag.v24i3.1720

Doerr, M., Gradmann, S., Hennicke, S., Isaac, A., Meghini, C., \& Van de Sompel, H. (2010). The europeana data model (edm). World Library and Information Congress: 76th IFLA General Conference and Assembly, 10-15.

Fernández-López, M. (1999). Overview of methodologies for building ontologies. IJCAI99 Workshop on Ontologies and Problem-Solving Methods: Lessons Learned and Future Trends, 430.

Fernández-López, M., Gómez-Pérez, A., \& Juristo, N. (1997). Methontology: from ontological art towards ontological engineering.

Gerstl, P., \& Pribbenow, S. (1996). A conceptual theory of part-whole relations and its applications. Data \& Knowledge Engineering, 20(3), 305-322.

Gruber, E., \& Smith, T. J. (2014). Linked open greek pottery. 42nd Annual Conference on Computer Applications and Quantitative Methods in Archaeology, 205-214.

Gruber, T. R. (1995). Toward principles for the design of ontologies used for knowledge sharing? International Journal of Human-Computer Studies, 43(5-6), 907928.

Guarino, N., \& Guizzardi, G. (2006). In the defense of ontological foundations for conceptual modeling. Scandinavian Journal of Information Systems, 18(1), 1.

Guarino, N., \& Welty, C. A. (2004). An overview of OntoClean. In Handbook on ontologies (pp. 151-171). Springer.

Lantow, B. (2016). OntoMetrics: Putting Metrics into Use for Ontology Evaluation. KEOD, 186-191.

Nong, J., Liang, C., and Xie, P. (2019). Research on government domain ontology construction method based on formal concept analysis. In 2019 IEEE International Conferences on Ubiquitous Computing \& Communications (IUCC) and Data Science and Computational Intelligence (DSCI) and Smart Computing, Networking and Services (SmartCNS), pages 230-235. IEEE.

Özacar, T., Öztürk, Ö., \& Ünalır, M. O. (2011). ANEMONE: An environment for modular ontology 
development. Data \& Knowledge Engineering, 70(6), 504-526.

Poveda-Villalón, M., Gómez-Pérez, A., and SuárezFigueroa, M.C. (2014). 'Oops! (ontology pitfall scanner!): An on-line tool for ontology evaluation', Int. J. Semantic Web Inf. Syst. IJSWIS, vol. 10, no. 2, pp. 734.

Ren, Y., Parvizi, A., Mellish, C., Pan, J. Z., Van Deemter, K., \& Stevens, R. (2014). Towards competency question-driven ontology authoring. European Semantic Web Conference, 752-767.

Roche, C., \& Papadopoulou, M. (2019). Mind the Gap: Ontology Authoring for Humanists. 1st International Workshop for Digital Humanities and Their Social Analysis (WODHSA)-Episode V: The Styrian Autumn of Ontology.

Ross, S. (2003). Towards a Semantic Web for Heritage Resources, vol. 3 of DigiCULT Thematic Issue. DigiCULT, Salzburg, May, 7-11.

Sabou, M., \& Fernandez, M. (2012). Ontology (network) evaluation. In Ontology engineering in a networked world (pp. 193-212). Springer.

Soergel, D. (1995). The art and architecture thesaurus (AAT): A critical appraisal. Visual Resources, 10(4), 369-400.

Suárez-Figueroa, M. C., Gómez-Pérez, A., \& FernándezLópez, M. (2012). The NeOn methodology for ontology engineering. In Ontology engineering in a networked world (pp. 9-34). Springer.

Suárez-Figueroa, M. C., Gómez-Pérez, A., \& FernandezLopez, M. (2015). The NeOn Methodology framework: A scenario-based methodology for ontology development. Applied Ontology, 10(2), 107-145.

Sure, Y., Staab, S., \& Studer, R. (2004). On-to-knowledge methodology (OTKM). In Handbook on ontologies (pp. 117-132). Springer.

Ushold, M., Gruninger, M. (1996). Ontologies: Principles, Methods and Applications. Knowledge Engineering Review, Vol. 11, $\mathrm{n}^{\circ}$ 2, June 1996. Also available from AIAI as AIAI-TR-191

Venu, S. H., Mohan, V., Urkalan, K., and Geetha, T. (2016). Unsupervised domain ontology learning from text. In International Conference on Mining Intelligence and Knowledge Exploration, pages 132-143. Springer

Winston, M. E., Chaffin, R., \& Herrmann, D. (1987). A taxonomy of part- whole relations. Cognitive Science, 11(4), 417-444.

Zouaq, A. and Nkambou, R. (2009). Enhancing learning objects with an ontology-based memory. IEEE Transactions on Knowledge and Data Engineering, 21(6):881-893.

冯先铭. (2002). 中国陶瓷 (上海古籍出版社).

霍华. (1989). 南京博物院藏瓷器标准化命名方法. 东南

文化, 198-201. 